\title{
Research on Dimension Adjustment Method of Variant Design
}

\author{
Hou Boqi, Li Xuemei,Wang Ke \\ School of Mechanical and Electrical Engineering, Guilin University of Electronic Technology, Guilin 541004 \\ Keywords: variant design; dimension dimension; dimension layout; size adjustment
}

\begin{abstract}
In order to study the problem of the dimension of the dimension of the model modified design. A geometric model for the layout of dimension dimension. Through the containment relationship between the dimensions of the relationship between the size of the resulting constraint relationship, on the basis of the creation of the size of the layout recognition algorithm, and the application validation. The size adaptive adjustment of the $3 \mathrm{D}$ variant design is realized.'
\end{abstract}

\section{Introduction}

Variant design is based on the specific needs of customers, to determine the design parameters of the product information,and according to the existing design as a template, the modified design of the main parameters of the model of the variant design, the variant method of similar products or parts will be generated. At present, many scholars both at home and abroad have carried on the research to the variant design. Xu Guoquan, etc[1]. Based on the design of the two parameters of the SolidWorks design and research, Wu Weiwei[2], et al. Based on the parametric design of mechanical product dimensional variant design and implementation, Xiao Lizhi, etc [3]-[6] proposed a method based on the parameters of the design method of dimensional containments, and so on. However, at present, the research on the dimension of the variant design process is less, and the size distribution of the variant is not effective. According to the above research, this paper mainly aims at solving the problem of the dimension of the model modified to solve the problem.

\section{A simplified model for the layout of dimension}

\section{1 dimension model}

As shown in Figure 1, a simplified model for 3D linear dimension. The dimension elements of the linear dimension model are the extraction point, the dimension normal vector, the dimension tangent vector, and the text box.

${ }^{1}$ Corresponding author : Hou Boqi,263998114@qq.com.

Project funding: Graduate education innovation project of Guilin University of Electronic Technology（GDYCSZ201440）. 


\subsection{The layout model of dimension}

This section puts forward a layout model of dimension variant design. In the past, the layout model of dimension is a kind of assumption that the dimension can not be arranged in the inner box, and the dimension can only be distributed around the box. At the same time, the specific shape and internal structure of the projection of the marking object are ignored. The dimension distribution of the object projection is shown in the 4 regions of the box. As shown in Figure 2, H1, H2, H3, H4.

However, in variant design, the model is changed, sometimes the size after modification will be in the model and this violated the above asumption that size cannot be arranged in the box. Then, a model of the size distribution of a rectangular bounding box is proposed, as shown in Figure 2. The model has the following characteristics: the change direction of the bounding box can only be marked along the 4 dimensions. The size parameters of the rectangular bounding box changes with the internal model.

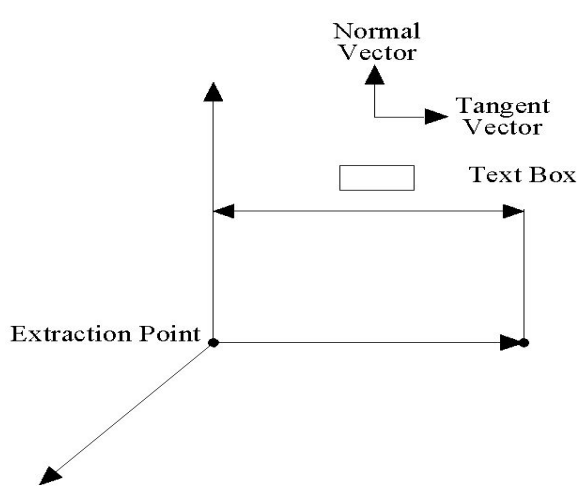

Figure 1. dimension model

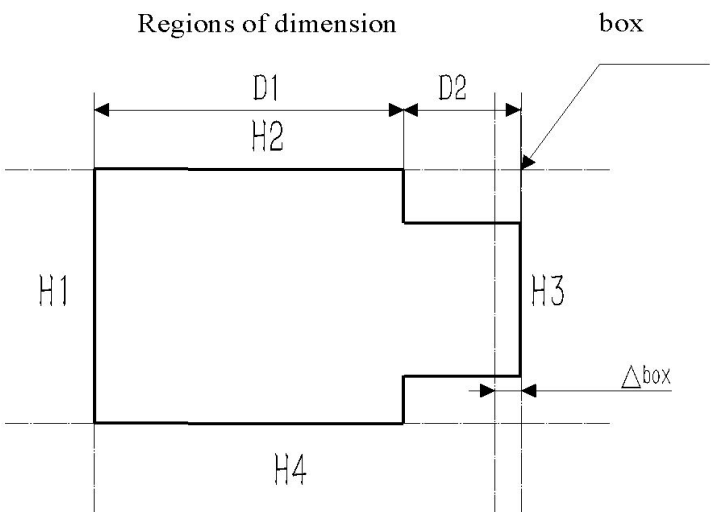

Figure 2. The layout model of dimension

\section{3 dimensional collection}

\section{1 dimension surface set partitioning}

American Standard ASME14.41 and the "three-dimensional CAD application technology specification" in the provisions: the geometry accuracy information of the marking plane should be parallel or perpendicular to the part coordinate system, in the GB/T24734 design model should include one or more model coordinate system, the general situation, the three dimension coordinate system can be in line with the corresponding parts or assembly or coordinate system by matrix transformation. According to the front two kinds of specifications can be drawn, the dimension is parallel or vertical to the basic dimension, feature labeling, user dimension. The realization method of $3 \mathrm{D}$ annotation is:

(1) a marking plane is determined in a three-dimensional model space;

(2) select the object, and then project the object onto the dimension;

(3) the marking is completed in accordance with the method of drawing a two-dimensional project icon;

The size of the size distribution is mainly for the dimensions of the same dimension. So we can divide the dimension according to its mark planes, and being in the same plane belongs to a same dimension. As shown in Figure 3, D1, D3, D4, belong to the same D2, D5 belongs to a label, D7, D6, belonging to the same label.The division method :of mark planes two non collinear nonzero vector can 
determine a plane, so you can get tangent vector and normal vector by the information data of size marking elements,then by means of vector operations, you can judge the planes all the dimensions belong to and classify them. The following is a vector operation method:

$$
\begin{aligned}
& \mathrm{Vij}=|\mathrm{Ni} \times \mathrm{Ti} \cdot \mathrm{Nj}|+|\mathrm{Ni} \times \mathrm{Ti} \cdot \mathrm{Tj}| \\
& \mathrm{Vij}=+
\end{aligned}
$$

$\mathrm{N}$ is normal vector , $\mathrm{T}$ is tangent vector , $\mathrm{i}(\mathrm{i}=1,2,3,4, . . \mathrm{n}, \mathrm{N},, \mathrm{j}=1,2,3,4, . . \mathrm{n}, \mathrm{N}, \ldots, \mathrm{Vij})$, $\mathrm{Vij}$ is the absolute value of two groups. When $\mathrm{Vij}=0$, the two dimensions are in the same plane. For example Figure 3 dimensions D1 and D2, D1 vector method for $\mathrm{N} 1$ cut vector T1 and the D2 normal vector is $\mathrm{N} 2$, and the tangent vector is $\mathrm{T} 2, \mathrm{~V} 12=+$. The $=0,=0,, \mathrm{~V} 12=0$, so the two dimensions belong to the same surface mark. For dimensions D1 and D5, D1 vector method for N1 cut vector T1 and D5 vector method for the $\mathrm{N} 5$ tangent vector for $\mathrm{T} 5, \mathrm{~V} 15=+$. The $=0, \neq 0, \mathrm{~V} 15 \neq 0$, so the two dimensions belong to the same surface mark.

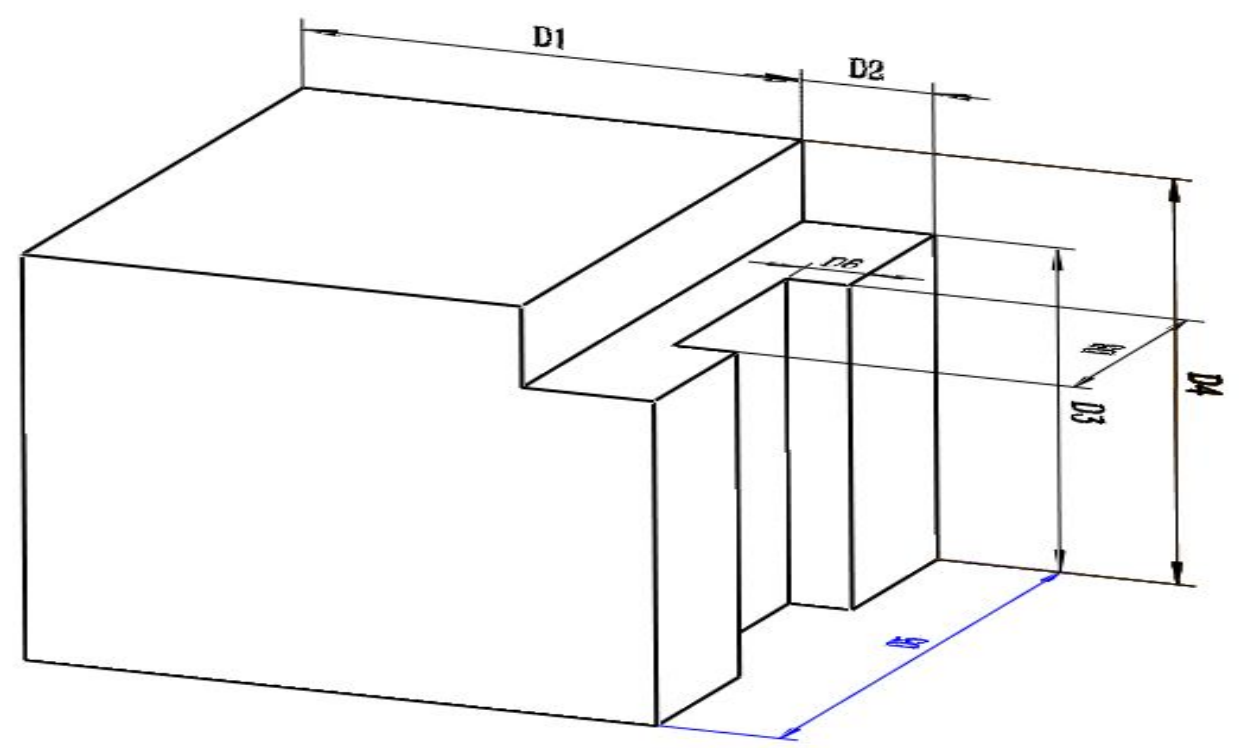

Figure 3.3D model

\section{2 size label face set}

As shown in Figure 2, the size of the Hi $(i=1,2,3,4)$ is based on the size of the layout model of the four regions to be divided, each dimension of the collection can be divided into four dimensions mark plane subset. Dimension face set partitioning method is mainly by size of vector method to determine the direction of division, collinear vectors and direction to be the same, as a group. As shown in Figure 3, D1, D3, D4, belong to the same D2, D1, D2, D3 and D4 are the same size, they are the same size, and the two are the same.

\section{Determination of position relationship between dimension of dimension}

\section{1 dimensional containment relationship}

For the same size of the size of the focus of the dimension, there is an inclusive relationship between the size of the dimension with it,you can rank the dimension, so as to lay the foundation for the reasonable layout of the dimension. There are mainly three kinds of relationships between the dimensions and containment relationship: (1) Fully containment relationship (2) The mutual non containment relationship(3)Semi containment relationship 


\section{2 size layout and size distribution}

The dimensions of the upper section of the containment relationship, although it can reflect the size of the approximate position between the size, but for the number of dimensions of the face is concentrated, and can not accurately express the relationship between the size of the. Therefore, it is necessary to take the size of containment relations as the basis of the size of the layout, to determine the size of the space between the constraints.

This paper is mainly to establish the integrated method of coordinate method, chain method, three kinds of commonly used size layout recognition methods were discussed.Coordinate method, the coordinate method used to label the parts that need to be set out from a reference.Chain, chain method is size followed by marking a chain, in mechanical manufacturing industry, chain method is often used to mark the distance between some of the same structure, ladder shaped parts size requirements very precise partition and combination items processing parts such as.Synthesis method, synthesis method of dimension chain is a comprehensive method and coordinate method, the label of parts size with comprehensive method. Thus, according to the function of the parts, processing method, shape structure and so on, need to use different forms of annotation dimension.

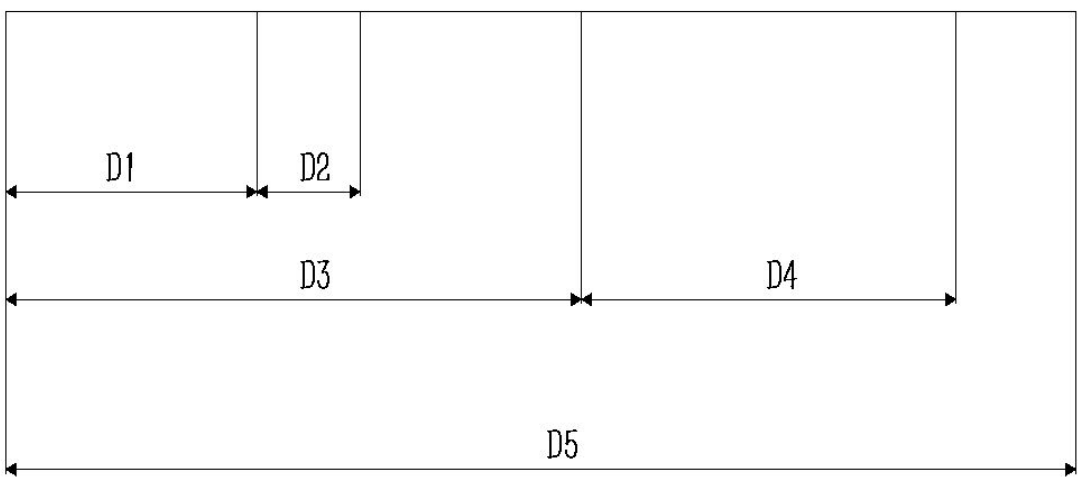

Figure 4 Synthesis method

The layout recognition algorithm is mainly based on the layout features of the layout, the layout characteristics of the layout.Coordinate method, the key factors are the following: (1) reference (2) dimension marking the distance to a constant (3) all dimensions are reference from near to far with reference to standard criterion. Key chain is the box marked the location of tangent vector collinear point. Two annotation method judgment principle: for two dimension if two adjacent size are not mutually inclusive, and the annotation box location point consisting of vector and cut collinear vectors both chain layout relations. If there is an inclusive relationship between the two dimensions, and there is a boundary between the two dimensions, the layout of the two is the coordinate method. For more than two dimensions. If they are not adjacent, it is not necessary to carry out the size of the layout of the judgment. According to II, if there is a size and in front of two dimensions and layout method of chain (coordinate placement method), three for the same chain layout method (coordinate placement method). In that subset size chain layout method belongs to all dimensions (coordinate layout). Finally, as shown in Figure 4, the overall layout of the integrated method is the comprehensive. 


\section{5 dimensional adjustment}

In the 3D software, due to the different parts of the different parts of the variant, the size may cause different changes in the layout of the size of the layout of the mess. Since the size distribution is determined, so we can adjust the size of the layout to be identified.

In the front, the size of the relationship and the size of the layout, the size of the space between the size of the location is determined by determining the size of the space between the constraints. As long as there is a size at the most appropriate location. The other dimensions can be adjusted by the correlation between them.According to change of process variant in size and is provided with a surrounding box change $\Delta$ box, the size change of the position of the text is $\Delta$ dim the following size adjusting method:For the size of a single dimension. The size of the need to adjust the amount of (boxdim).Dimensions of two or more than two dimensions. Determine both subsumption relations and distribution relations, (1) if there is a dimension of the $\Delta \operatorname{dim}=\Delta$ box, is the size does not move, according to the size of the position as a moving reference other dimensions. (2) if all sizes of $\Delta \operatorname{dim}$ and $\Delta$ box, Then look for a dim=0 size, the size and quantity of mobile, mobile as a box.By the above method, at least one size can be moved to the right place, for many dimensions, because of the containment of the relationship and the layout of the determination, the other dimensions of the need to move the location is also easy to get.

\section{6 conclusions}

Through the analysis of the characteristics of size distribution, the size distribution model is established, and the containment of the dimension is distinguished by the characteristics of dimension. All dimensions are related to each dimension, and the location of each dimension is determined by the containment relationship. After the final type of the size of the size of the characteristics, to find an optimal position of the size, the final overall adjustment. Finally, it proves that it is proved by the example

\section{References}

[1]XU Guo-quan, HUANG Zhi-chao.Research on Parametric Design in Its Further Development Based on SolidW orks.Journal of Machine Design and Research. 2007,23(1):68-87.

[2]Wu Weiwei,Tang Renzhong,Hou Liang,Wang ZhengxiaoResearch \&Realization on the Variant Design of Mechanical Products Based on Parametric Techniques. China Mechanical Engineering.2005,16(3):218-222

[3] XIAO Li-Zhi, HUANG Mei-Fa, LIU Fu-Yun.. Parametric Design Method of Dimensional Tolerance Based on SolidWorks.Computer Systems \& Application,2010,20(19):210-213.

[4]Deng Xiaolin, Liu Fuyun. Design method of part variant design based on parametric technology, mechanical design and research, 2008, 24 (4):47-49.

[5]Fuyun Liu, Sujing Song and Xiaolin Deng. Research on evolving rule of part relation net work of product family. Int. J. Materials and Structural Integrity, Vol.1, No.4, 2008.

[6]Liu Fuyun, Deng Xiaolin. Research on the design method of product dimension parameter modification and variant design.EC, 2008, 14 (11): 2092-2096. 